\title{
Kartagener Syndrome: A Rare Genetic Disorder
}

\author{
Shakya $\mathrm{K}^{1}$ \\ 'Laliptpur, Nepal
}

\begin{abstract}
Kartagener Syndrome is a rare autosomal recessive disorder consisting of triad of sinusitis, bronchiectasis and situs inversus with dextrocardia. It is the subset of disorder called primary ciliary dyskinesia in which the cilia have abnormal structure and/or function resulting in multisystem diseases of various severity. Clinical manifestations include lifelong, chronic upper and lower respiratory tract diseases secondary to ineffective mucociliary clearance. Early diagnosis and management of chest infections can prevent irreversible damage to lungs and prevent potential lifelong complications. This case report is on a patient who presented with long standing history of sinusitis, bronchiectasis and on examination situs inversus with dextrocardia.
\end{abstract}

Key Words: bronchiectasis, dextrocardia, kartagener syndrome, primary ciliary dyskinesia, situs inversus

\section{INTRODUCTION}

Primary ciliary dyskinesia (PCD) is an autosomal recessive disorder with an incidence of 1 in 2000030000. ${ }^{1}$ Ultrastructural defects are present in cilia and spermatozoa. Ineffective mucociliary clearance predisposes to recurrent sinopulmonary infections, irreversible bronchopulmonary damage leading to cor pulmonale. In addition, females can present with subfertility while males demonstrate infertility secondary to immotile spermatozoa. ${ }^{2,3}$ Kartagener syndrome is a subset of this disorder and is observed in about 50 percent of patients. ${ }^{4}$

This patient presented with clinical signs and symptoms of bronchiectasis and chronic sinusitis with situs inversus. The importance of diagnosing this rare disorder lies in the fact that deterioration in lung function can largely be prevented by early and appropriate respiratory care.
Moreover, this syndrome might present with atypical clinical signs and symptoms due to reverse positioning of viscera and can bring potential diagnostic dilemma and surgical errors if not recognized in the early course of treatment.

\section{CASE REPORT}

A 17 year old female presented with a long standing history of recurrent cough, production of copious amount of thick purulent sputum with occasional hemoptysis, on and off chest pain and mild shortness of breath on exertion. She had excessive, thick nasal discharge with persistent congestion of nose and headache with pain over cheek region which was exacerbated during winter seasons. She also complained of long standing inability to perceive smell. She gave past history of multiple episodes of hospital admissions for pneumonia for which she was successfully treated with antibiotics.

\author{
Correspondence: \\ Dr. Kunjan Shakya \\ G.P.O. Box 8975, E.P.C. 957 \\ Lalitpur, Nepal. \\ Email: kunjan_shakya@hotmail.com
}


There was no history of similar presentation in her first and second degree relatives on both maternal and paternal sides and consanguineous marriage among her parents and no history of tuberculosis or smoking and exposure to it. Her mother had a past history of tuberculosis for which she was treated successfully. Menarche was at the age of 15 years with irregular cycle and dysmenorrhoea for last two years.

There was no cardiac dullness on left precordial area of the chest on percussion; instead it was elicited on the right parasternal area of the chest with maximal cardiac impulse on the fifth intercostal space. The heart sounds were normal without any murmur. There were crepitations and wheezes over bilateral lung fields. Hepatic dullness was elicited on the left side and tympanic note on the right side. The nasal mucosa was pale and swollen with nasal septum deviation over the left side. Bilateral maxillary sinuses tenderness could be elicited.

Chest X-ray was done while paying special attention on labeling the sides on plates which revealed dextrocardia, with the cardiac apex pointing towards the right, the arch of aorta and fundic gas shadow of the stomach noted on the right side (Figure 1). The hemi diaphragm was raised over the left side.

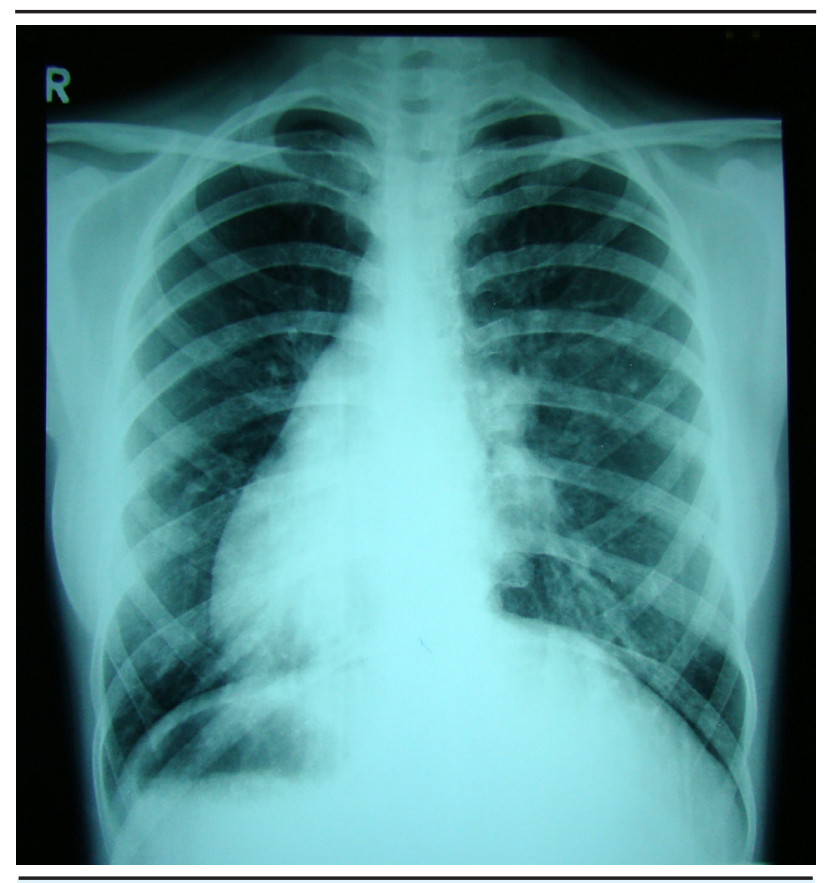

Figure 1. Chest X-ray image revealing dextrocardia

High resolution computed tomography (HRCT) was done to visualize the bronchiectatic changes and the extent of involvement which showed dilated, fluid filled bronchi with peribronchial thickening and a ring like appearance over the bilateral lower lobes, left upper lobe and lingula (Figure 2). The lingula of the lung and heart was noted on the right side.

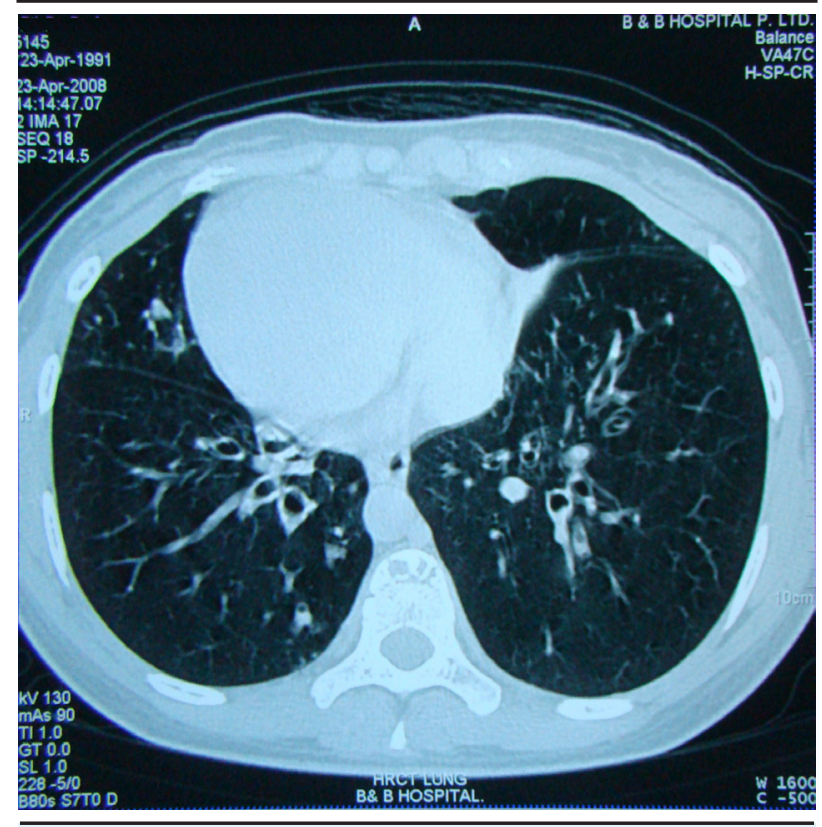

Figure 2. HRCT image showing bronchiectatic changes

Computed tomography (CT) scan of the paranasal sinuses showed diffuse hypodense mucosal thickening involving all the paranasal sinuses with sparing of few ethmoidal cells (Figure 3 ). Air fluid levels were noted in bilateral maxillary sinuses and deviation of nasal septum over the left side with hypertrophy of middle and inferior turbinates. Bilateral osteomeatal complexes were obliterated due to mucosal thickening.

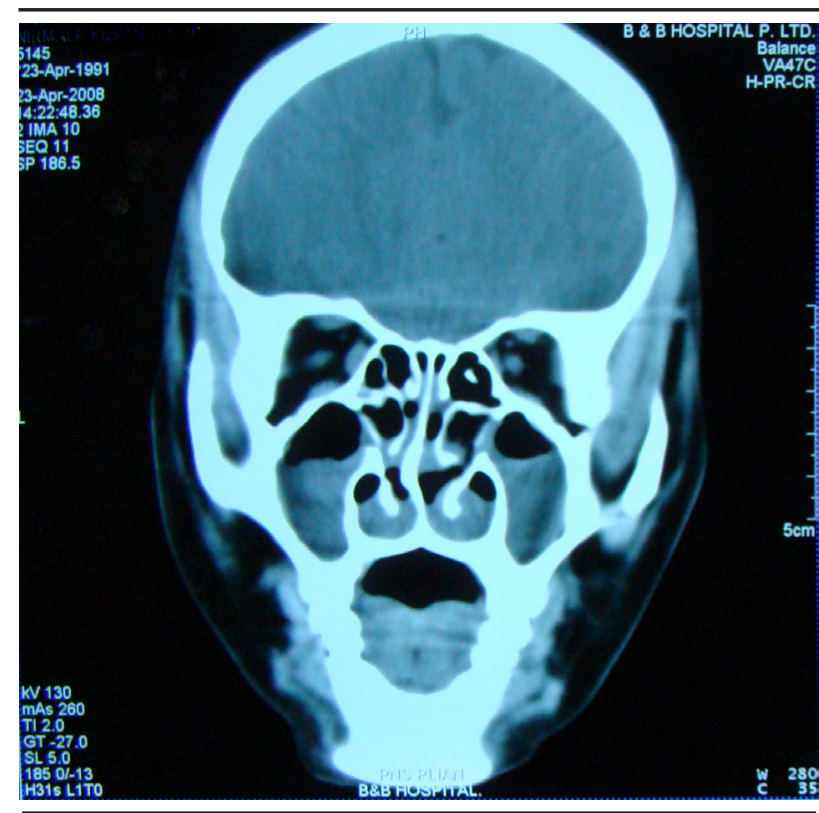

Figure 3. CT scan of the paranasal sinuses showing diffuse hypodense mucosal thickening

Ultrasonography of the abdomen showed the reversal of positions of the viscera, visibly spleen, stomach, splenic flexure of colon and sigmoid colon over the 
Shakya. Kartagener Syndrome: A Rare Genetic Disorder

right side and liver, hepatic flexure of colon over the left side.

Electrocardiography (ECG) was done to rule out any conduction abnormalities of the heart or see for any hypertrophic changes due to possible pulmonary hypertension secondary to bronchiectatic changes of the lungs (Figure 4,5). On conventional positioning of leads, ECG showed inverted $P$ waves in lead I (Figure 4). The normal appearances of standard leads II and III were interchanged. There was poor progression of QRS complexes with right axis deviation. However, after reversal of limb leads and precordial leads, it showed normal wave pattern with sinus tachycardia without any conduction abnormalities or any other significant abnormality (Figure 5).

Echocardiography was done which did not reveal any anatomical abnormality of heart except for dextrocardia. The Tuberculin Skin Test was negative. The saccharin test of mucociliary clearance gave a time of 47 minutes (normal result less than 15 minutes). Smell test was done with coffee and isopropyl alcohol on each nostril separately. This test showed inability to perceive smell bilaterally.

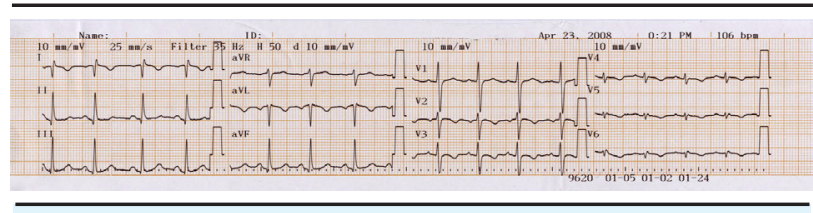

Figure 4. ECG with conventional positioning of leads showing inverted $\mathrm{P}$-waves in lead I

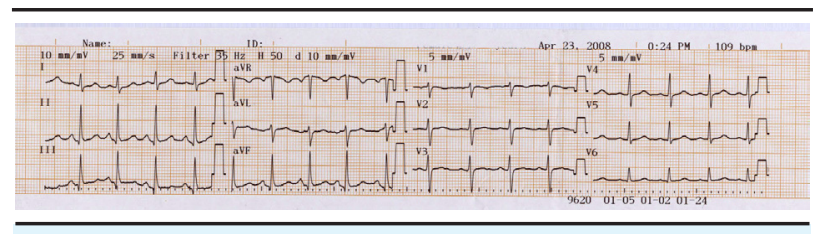

Figure 5. ECG with reversal of limb and precordial limb leads showing normal wave pattern with sinus tachycardia without any conduction abnormalities or any other significant abnormality

\section{DISCUSSION}

Siewert was the first person to describe the combination of situs inversus, chronic sinusitis and bronchiectasis in 1904. ${ }^{5}$ However, Manes Kartagener first recognized this triad as a distinct congenital syndrome in 1933 and described it in detail, hence it bears his name. ${ }^{5}$ It is associated with mutations in several genes, but mainly affecting DNAI1 on chromosome 9p21-p13, DNAH5 on chromosome 5p15-p14 and DNAH11 on chromosome $7 p 21$ which results in numerous defects including structural abnormalities of the dynein arms, radial spokes, and microtubules of the cilia. ${ }^{6}$ Afzelius pointed out that electron microscopy shows different kinds of ultrastructural defects of cilia and sperm tails in the immotile cilia syndrome. Since the cilium is constructed of some 100 different polypeptides, there is ample room for genetic heterogeneity. ${ }^{7}$ Cilia are normally present on the ventral surface of the primitive node and may be involved in left-right patterning during gastrulation. ${ }^{8}$

Impaired host defense mechanisms due to ciliary dysfunction are involved in the predisposition to recurrent infections. Patients typically present with persistent or recurrent cough and purulent sputum production. Hemoptysis occurs in 50 to $70 \%$ of cases and can be due to bleeding from friable, inflamed airway mucosa. However, other causes of hemoptysis should be ruled out first before attributing it to the above cause. Dyspnea or wheezing generally reflects either widespread bronchiectasis or underlying chronic obstructive pulmonary disease. Patients with severe, diffuse disease, particularly those with chronic hypoxemia, may have associated cor pulmonale and right ventricular failure. In patients with extensive disease, chronic hypoxemia and cor pulmonale may indicate the need for long-term supplemental oxygen.

Isolated dextrocardia is almost always associated with other cardiac anomalies, which are often serious. When accompanied by situs inversus, serious cardiac malformations are less common, but these and anomalies of skeleton, spleen and other viscera have been described. ${ }^{9-11}$ However in this patient, apart from complete situs inversus, no other abnormalities were noted on echocardiography and ultrasonogram. Corneal abnormalities, headaches, and a poor sense of smell have been attributed to the primary defect in the immotile cilia syndrome. ${ }^{12}$ Headache and anosmia are also present in this patient.

Some people with PCD feel stigmatized and isolated due to their condition. ${ }^{13}$ Since half of individuals with PCD have situs inversus, a physical but concealable bodily abnormality, decisions have to be made about revealing this atypicality to others and they have difficulty in doing so. ${ }^{14}$ They also particularly attempt to hide coughing and nose-blowing. The low prevalence of PCD means there would be a paucity of public knowledge, which leads to lack of support and understanding to them. ${ }^{13}$

Early diagnosis and treatment in this syndrome is very important to prevent the deterioration of the lung function and the morbidity caused due to upper and lower respiratory tract infections. There are currently no reliable noninvasive diagnostic methods for this disease and the correct diagnosis is often delayed many years, possibly causing irreversible pulmonary damage. ${ }^{15} \mathrm{~A}$ study led by Lundberg et al. has found considerably low level of nasal Nitric Oxide ( $98 \%$ 
Shakya. Kartagener Syndrome: A Rare Genetic Disorder

reduced) in patients with PCD compared to agematched controls and hence suggested that Nitric Oxide measurements (which are noninvasive and can easily be performed even in infants) could be of help in the early diagnosis. ${ }^{16}$ With improved quality of care, survival of these patients are not affected. The case of a woman who was documented with this syndrome at the age of 72 years fairly suggests that this syndrome is compatible with full life span. ${ }^{17}$
Kartagener Syndrome can have variable presentations and severity due to its multisystem involvement and reversal of viscera. Early diagnosis and treatment is important to prevent long term sequel and morbidity associated with it. A noninvasive screening test should be devised for its early detection. Genetic counseling, social, psychological and fertility issues should be addressed once it is diagnosed and help these patients to live with Kartagener Syndrome without morbidity and in a dignified way.

\section{REFERENCES}

1. Blouin JL, Meeks M, Radhakrishna U, Sainsbury AJ, Gehring C, Duriaux Saïl G et al. Primary ciliary dyskinesia: a genomewide linkage analysis reveals extensive locus heterogeneity. Eur J Hum Genet 2000 Feb; 8(2):109-118.

2. Afzelius BA, Mossberg B. Immotile cilia syndrome, including Kartagener syndrome. In: Scriver $\mathrm{CR}$ et al., (editor). The Metabolic Basis of Inherited Disease, 6th ed. New York : McGraw-Hill, 1989.

3. Arge E. Transposition of the viscera and sterility in men. Lancet 1960 Feb 20; 1(7121):412-414.

4. Rott HD. Kartagener's syndrome and the syndrome of immotile cilia. Hum Genet 1979; 46: 249-261.

5. Bent JP, Olearczyk M. Kartagener Syndrome. emedicine[Internet]. Jan 3, 2007 [cited June 3, 2008]. Available from: URL:http://www.emedicine.com/med/topic1220. htm

6. Kartagener Syndrome. Online Mendelian Inheritance in Man, OMIM (TM). Johns Hopkins University, Baltimore, MD [Internet]. MIM Number: \#244400. March 17, 2008 [cited June 3, 2008]. Available from: URL:http://www.ncbi.nlm.nih. gov/omim/

7. Afzelius BA, Eliasson R. Flagellar mutants in man: on the heterogeneity of the immotile-cilia syndrome. J. Ultrastruct. Res. 69: 43-52, 1979. PubMed ID : 501788

8. Sadler TW. Langman's medical embryology. 9th ed. Philadelphia(PA): Lippincott Williams and \& Wilkins; 2004. p. 75.

9. Putschar WGC, MAnion WC. Congenital absence of the spleen and associated anomalies. Amer J Clin Path 1956; 26: $29-470$.
10. Torgersen J. Anomalies of the spine in anomalies of viscera and constitution. Acta Radilo [Diagn] (Stockholm) 1948; 29:311-320.

11. Merklin RJ, Varano NR. Situs inversus and cardiac defects: a study of 111 cases of reversed asymmetry. J Thorac Cardiovasc Surg 1963; 45: 334-342.

12. Neustein HB, Nickerson B, $\mathrm{O}^{\prime}$ Neal M. Kartagener's syndrome with absence of inner dynein arms of respiratory cilia. Am Rev Resp Dis 122: 979-981, 1980.

13. Whalley S, McMannus IC. Living with primary ciliary dyskinesia: a prospective qualitative study of knowledge sharing, symptom concealment, embarrassment, mistrust, and stigma. BMC Pulm Med [Internet] 2006; 6: 25.Oct 13.2006 [cited June 3, 2008]. Available from: URL:http://www. pubmedcentral.nih.gov/articlerender.fcgi?tool=pmcentrez\& artid=1635565

14. McManus IC, Stubbings GF, Martin N. Stigmatization, physical illness and mental health in primary ciliary dyskinesia. J Health Psychol 2006; 11: 467-482.

15. Levison H, Minsdorff CM, Chao J, et al. Pathophysiology of the ciliary motility syndromes. Eur J Respir Dis 1983; 64 (Suppl. 127): 102-116.

16. Lundberg JON, Weitzberg E, Nordvall SL, Kuylenstierna R, Lundberg JM, Alving K. Primarily nasal origin of exhaled Nitric Oxide and absence in Kartagener's syndrome. Eur Respir J 1994; 7: 1501-1504.

17. Miller RD, Divertie MB. Kartagener syndrome. Chest 1972; 62: 130-135. 